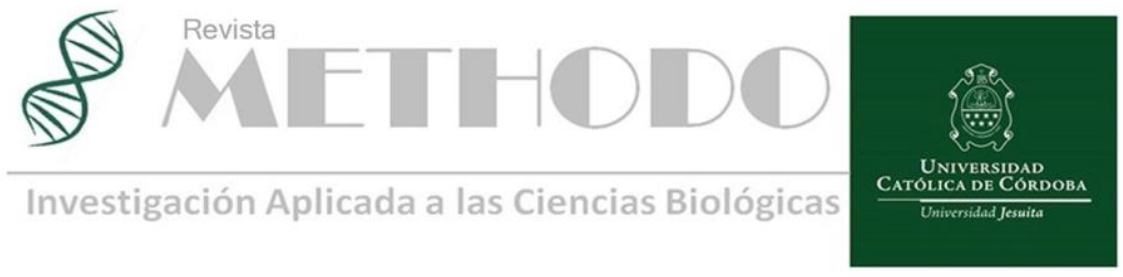

ARTICULO ORIGINAL Rev. Methodo 2021;6(3):121-126 https://doi.org/10.22529/me.2021.6(3)04

Recibido 28 Feb. 2020 | Aceptado 30 Jul. 2020 | Publicado 02 Jul 2021

\title{
Etiología y perfil de sensibilidad en infecciones urinarias en pediatría
}

\section{Etiology and sensitivity profile in urinary tract infections in pediatrics}

\author{
Gabriela Guidi $^{1}$ (iD), Marina Teresita Bottiglieri², Agostina Zucotti ${ }^{2}$ \\ 1 Universidad Católica de Córdoba, Facultad de Ciencias de la Salud, Clínica Universitaria Reina Fabiola, Servicio de Pediatría. \\ 2 Universidad Católica de Córdoba, Facultad de Ciencias de la Salud, Clínica Universitaria Reina Fabiola, Servicio de Microbiología. \\ Correspondencia: Gabriela Guidi. E-mail: gabiguidi91@gmail.com.
}

\section{Resumen}

INTRODUCCIÓN: La infección del tracto urinario (ITU) se define como la invasión, colonización y proliferación bacteriana del tracto urinario. Se sabe que puede ser producida por diferentes microorganismos patógenos, pero actualmente existen pocos datos en relación a la etiología y resistencia a antimicrobianos en esta infección. Se sabe que esta patología es la segunda causa más frecuente de infecciones bacterianas en niños, y que es la responsable de gran número de consultas e internaciones a diario en el servicio de Pediatría.

OBJETIVO: Describir el agente causal más prevalente de infecciones del tracto urinario en pacientes pediátricos y el perfil de sensibilidad antibiótica durante el periodo de enero 2015 hasta diciembre 2017 en Clínica Universitaria Reina Fabiola.

MATERIALES Y METODOS: Estudio observacional, retrospectivo, descriptivo. Se realizó una recolección de datos, desde el sistema de microbiología, de pacientes con diagnóstico de ITU (basándonos en resultado positivo de urocultivo) que consultaron en el Servicio de Pediatría de la Reina Fabiola en el periodo de enero 2015 a diciembre 2017. Se evaluaron las siguientes variables: sexo, edad, resultado microbiológico de urocultivo y de antibiograma. Las variables se describieron con $\mathrm{N}(\%)$ en caso de variables categóricas y medidas de posición y dispersión en caso de variables continuas.

RESULTADOS: Se procesaron 3618 urocultivos resultando positivos 867 (24\%), de los cuales 539 se incluyeron en el presente estudio por contar con datos completos. Se demostró una predominancia en el sexo femenino $(83,8 \%$ ) y el grupo etario donde predominaron las ITU fue entre el año y los 5 años N: 259 (48\%). El microorganismo más frecuente fue la Escherichia coli, 441/539 81\%), seguida por Proteus mirabillis (49/539 9\%) y Klebsiella pneumoniae (12/539 2\%). La Escherichia coli fue sensible a nitrofurantoina, cefazolina, ciprofloxacina, ampicilina sulbactam $(83,2 \%)$ y en menor porcentaje a trimetropina sulfametoxazol, siendo resistente a ampicilina. El Proteus mirabillis y la Klebsiella pneumoniae presentaron un amplio perfil de sensibilidad.

CONCLUSIÓN: Este estudio permitió conocer que las infecciones urinarias en nuestro medio en la edad pediátrica son más frecuentes en el sexo femenino y entre el año y los 5 años de vida. El agente etiológico más común es la Escherichia coli, seguido por el Proteus mirabillis y la Klebsiella pneumoniae, los cuales presentan una alta sensibilidad antibiótica a cefazolina, ciprofloxacina y ampicilina sulbactam.

Palabras claves: infección urinaria, bacteria, sensibilidad antibiótica, pediatría. 


\begin{abstract}
INTRODUCTION: Urinary tract infection (UTI) is defined as the bacterial invasion, colonization and proliferation of the urinary tract. It can be produced by different pathogenic microorganisms, but there are currently few data regarding the etiology and antimicrobial resistance in this infection. It is known that this pathology is the second most frequent cause of bacterial infections in children, and that it is responsible for a large number of daily consultations and hospitalizations in the Pediatric services.

OBJECTIVE: To describe the most prevalent causative agent of urinary tract infections in pediatric patients and the antibiotic sensitivity profile during the period from January 2015 to December 2017 at Clínica Universitaria Reina Fabiola.

MATERIALS AND METHODS: This is an observational, retrospective and descriptive studyThe following variables were evaluated: sex, age, microbiological result of urine culture and antibiogram. The variables were described with $\mathrm{N}(\%)$ in case of categorical variables and measures of position and dispersion in case of continuous variables.

RESULTS: 3618 urine cultures were processed, with 867 (24\%) positive, of which 539 were included in this study due to complete data. Predominance in the female sex $(83.8 \%)$ was demonstrated and the age group where the UTIs predominated was between 1 year and 5 years $\mathrm{N}=259(48 \%)$. The most frequent microorganism was Escherichia coli, 441/539 81\%), followed by Proteus mirabillis (49/539 9\%) and Klebsiella pneumoniae (12/539 2\%). Escherichia coli was sensitive to nitrofurantoin, cefazolin, ciprofloxacin, ampicillin sulbactam $(83.2 \%)$ and trimetropin sulfamethoxazole to a lesser extent, being ampicillin resistant. Proteus mirabillis and Klebsiella pneumoniae had a broad sensitivity profile.

CONCLUSION: This study showed that urinary infections in our pediatric age environment are more frequent in females between 1 year and 5 years old. The most common etiologic agent is Escherichia coli followed by Proteus mirabillis and Klebsiella pneumonia. All of them showing a high antibiotic sensitivity to cefazolin, ciprofloxacin and ampicillin sulbactam.
\end{abstract}

KeyWords: Urinary tract infection, bacteria, antibiotic sensitivity, pediatrics.

\section{Introducción}

La infección del tracto urinario (ITU) se define clásicamente como la invasión, colonización y proliferación bacteriana del tracto urinario, que puede comprometer desde la vejiga hasta el parénquima renal ${ }^{1}$. Se puede presentar desde una bacteriuria asintomática, hasta las formas sintomáticas como la cistitis, pielonefritis aguda, nefronía lobar aguda y la formación de abscesos renales. La sintomatología que acompaña a dicha patología es variable, siendo desde síntomas urinarios bajos hasta fiebre asociada a dolor lumbar, rechazo alimentario, irritabilidad entre otros $^{2}$.

Esta patología es la segunda causa más frecuente de infección bacteriana en niños, luego de las infecciones respiratorias. Hacia los 7 años de edad entre $8-10 \%$ de las niñas y el $2-3 \%$ de los niños habrán tenido una ITU sintomática. Si bien la prevalencia es mayor en mujeres que en varones, en los primeros años de vida la diferencia entre sexos es menor (relación 1: 1), siendo en los primeros 3 meses más frecuente en varones produciéndose un incremento progresivo, con predominio en mujeres a partir del año de vida ${ }^{3}$. Se consideran factores de riesgo las anomalías del tracto urinario, la uretra corta, la fimosis en lactantes varones, la disfunción vesical, el estreñimiento y la infestación por oxiuros, además de la instrumentación de la vía urinaria, la vejiga neurogénica y el reflujo vesicoureteral ${ }^{4-5}$.

Los agentes etiológicos más frecuentes son bacterias, pero también pueden ser en menor medida virus, hongos, y parásitos. Escherichia coli es el primer agente etiológico de ITU tanto en niños como en adultos, seguido por Proteus mirabilis, Klebsiella pneumoniae, otras enterobacterias, Staphylococcus aureus, Enterococccus spp., y Pseudomonas aeruginosa. Con excepción de los tres primeros microorganismos citados, el resto está principalmente asociado a infecciones relacionadas al cuidado de la salud, pacientes inmunocomprometidos, malformaciones de la vía urinaria, vejiga neurogénica e instrumentación urológica siendo muy poco frecuente como agentes causales de ITU adquiridas en la comunidad. En recién nacidos es posible encontrar Streptococcus agalactiae ${ }^{6-7}$

El diagnostico de ITU se realiza a través de la recolección de una muestra de orina para cultivo, el cual se considerado positivo al aislar un microorganismo patógeno, asociado a respuesta inflamatoria. Dependiendo de la forma de recolección de la muestra de orina, varían los puntos de corte para ser considerada positiva. Siempre en contexto de reacción inflamatoria, se

Revista Methodo: Investigación Aplicada a las Ciencias Biológicas. Universidad Católica de Córdoba. Jacinto 
considera una bacteriuria significativa el aislamiento de más de 102 UFC/ml en el cultivo de orina obtenida mediante punción suprapúbica, más de $104 \mathrm{UFC} / \mathrm{ml}$ en cultivo de una muestra procedente de cateterismo vesical y más de 105 $\mathrm{UFC} / \mathrm{ml}$ en muestra recolectada al asecho ${ }^{8}$.

El diagnóstico temprano y el abordaje terapéutico correcto son de fundamental importancia a fin de evitar la formación de cicatrices en el parénquima renal. Entre las secuelas tardías relacionadas con las cicatrices renales figuran: hipertensión arterial, proteinuria, lesión renal e incluso insuficiencia renal crónica?.

El tratamiento antibiótico empírico inicial está indicado en las infecciones urinarias hasta obtener el resultado del urocultivo, el que nos permitirá colocar el tratamiento específico y apropiado para dicha infección, que variará según la edad, sexo, microorganismo asociado y tipo de infección. Para que un antibiótico pueda ser utilizado en forma inicial no debe tener una tasa de resistencia superior a 10-20\%. La resistencia bacteriana ha ido en aumento por el uso continuo e inapropiado de antibióticos de amplio espectro que conlleva el aumento de la flora multirresistente ${ }^{9}$

\section{Objetivo}

Describir el agente causal más prevalente de infecciones del tracto urinario en pacientes pediátricos en nuestro medio y su relación con edad y sexo.

Describir el perfil de sensibilidad antibiótica de microrganismos causantes de infección tracto urinario en pacientes pediátricos en nuestro medio

\section{Material y métodos}

Pacientes en edad pediátrica ( 1 mes a 15 años) que consultaron en el servicio de pediatría de la Clínica Universitaria Reina Fabiola de forma ambulatoria (guardia pediátrica y consultorio externo). Clínica Universitaria Reina Fabiola. Servicio de pediatría y microbiología. Desde el 1 enero 2015 hasta 31 diciembre 2017.

Criterios de inclusión:

- Pacientes de 1 mes a 15 años de edad.

- Urocultivo positivo (independiente de toma de muestra).

Se recolectaron datos, desde el sistema del Servicio de Microbiología, de urocultivos positivos de pacientes que consultan en el Servicio de Pediatría de la Reina Fabiola en el periodo de enero 2015 a diciembre 2017. La recolección de la muestra se llevó a cabo, previa higiene con agua y jabón neutro, por la técnica de chorro medio. En pacientes sin control de esfínteres (lactantes y niños pequeños) se utilizó recolección al acecho y cateterismo vesical en caso excepcional. La muestra se procesó dentro de las dos horas de recolección o fue conservada a $4^{\circ} \mathrm{C}$ hasta 24 hs. Se consideró respuesta inflamatoria (RI) cuando se obtuvo un recuento de leucocitos PMN de más de 5/campo al examen de sedimento urinario o más de $10 / \mathrm{mm} 3$ de una gota de orina sin centrifugar por el recuento de cámara de Neubauer. Se consideró urocultivo positivo aquella muestra que presento una RI y cultivo entre 1.000 a $100.000 \mathrm{UFC} / \mathrm{ml}$ de orina de un solo microorganismo en la muestra, dependiendo la clínica del paciente. El cultivo de orina se realizó con ansa calibrada en agar CLED (Cisteína Lactosa Electrolito Deficiente) y se incubo por 24 horas en aerobiosis a $35-37$ oC en microerofilia durante 24 hs., con la finalidad de investigar microorganismos más exigentes se sembró en agar chocolate por 48hs. La identificación bioquímica de los distintos mioorganismos se realizó según técnicas citadas en la bibliografía internacional.

\section{Variables estudiadas}

- Variables independientes: sexo, edad.

- Variables dependientes: resultado icrobiológico de urocultivo.

Resultado de antibiograma.

\section{Consideraciones éticas}

El estudio se realizará cumpliendo con las normativas de la declaración de Helsinki y Buenas prácticas clínicas de ANMAT y Ley provincial N. ${ }^{\circ}$ 9694. Los autores declaran no tener conflictos de intereses. Se aseguró la protección de datos personales de los pacientes según la ley 253226. No requiere consentimiento informado.

\section{Resultados}

Se analizaron 3618 urocultivos pertenecientes a pacientes pediátricos (de un mes de vida a 15 años) que consultaron de forma ambulatoria (guardia pediátrica y consultorio externo) por el Servicio de Pediatría de la Clínica Universitaria Reina Fabiola. De los urocultivos analizados resultaron positivos 867 (24\%), de los cuales 539 se incluyeron en el presente estudio por contar con datos completos. En cuanto al género se encontró que $452(83,8 \%)$ fueron mujeres y $87(16,2 \%)$ fueron varones. La distribución etaria de los pacientes fue de 20 $(3,7 \%)$ entre el mes y los tres meses, $73(13,5 \%)$ entre los tres meses y un año, 259 (48\%) entre el año y los 5 años y 189 (35\%) entre los 5 y 15 años. En todos los grupos etarios predominaron las ITU en mujeres (Figura 1). 


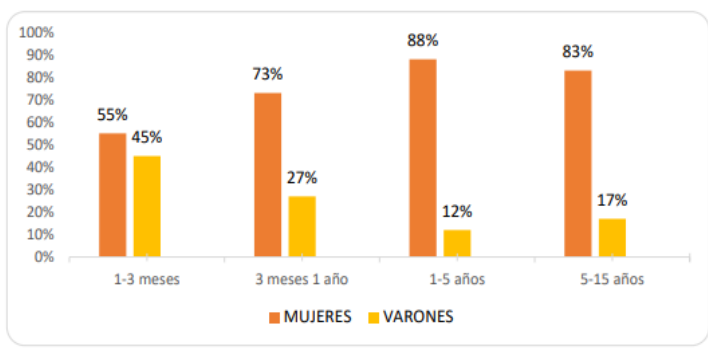

Figura 1. Infecciones urinarias en pediatría distribución del sexo según grupo etario.

El agente causal más frecuente fue la Escherichia coli $(441 / 539 ; 81 \%)$, seguida por Proteus mirabillis $(49 / 539 ; 9 \%)$ y Klebsiella pneumoniae $(12 / 539 ; 2 \%)$ (Figura 2).

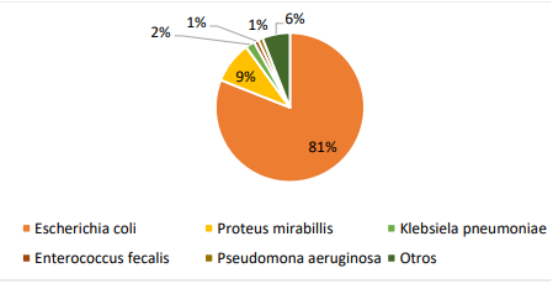

Figura 2. Infecciones urinarias en pediatría. Distribución según agente etiológico.

La Escherichia coli predominó en todos los grupos etarios, mientras que la Klebsiella

pneumoniae fue el segundo agente más frecuente en el grupo de 1 a 3 meses y de 3

meses a un año, y el Proteus mirabillis en el grupo de 1 a 5 años y de 5 a 15 años

(Figura 3).

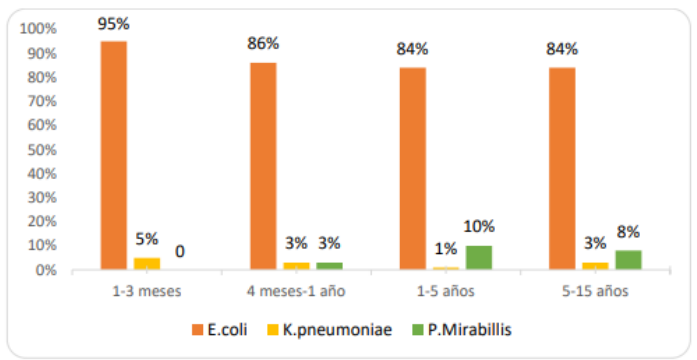

Figura 3. Infecciones urinarias en Pediatría. Microrganismo más frecuente según grupo etario.

En cuanto al agente etiológico según sexo, la Escherichia coli continúo predominando en ambos géneros (Figura 4).

Al analizar la sensibilidad microbiana, se observó que la Escherichia coli fue sensible a nitrofurantoína $(99,1 \%)$, cefazolina $(97,1 \%)$, ciprofloxacina $(90,6 \%)$, ampicilina sulbactam $(83,2 \%)$ y trimetropina sulfametoxazol $(60,7 \%)$, mostrando resistencia a ampicilina (62,8\%) (Figura 5 y Tabla 1).

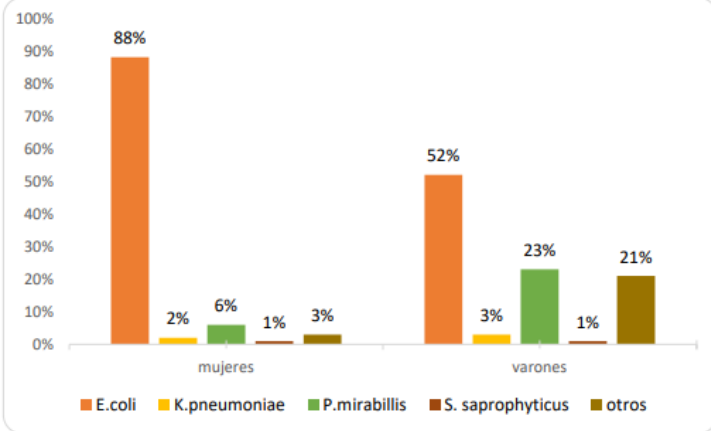

Figura 4. Infecciones urinarias en Pediatría. Agente etiológico según sexo.

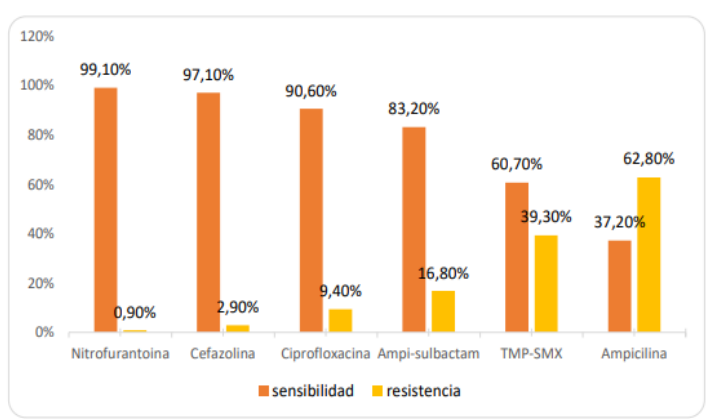

Figura 5. Infecciones urinarias en Pediatría. Perfil de sensibilidad de E. coli.

Tabla 1. Infecciones urinarias en Pediatría. Perfil de sensibilidad antibiótica de los agentes etiológicos más frecuentes.

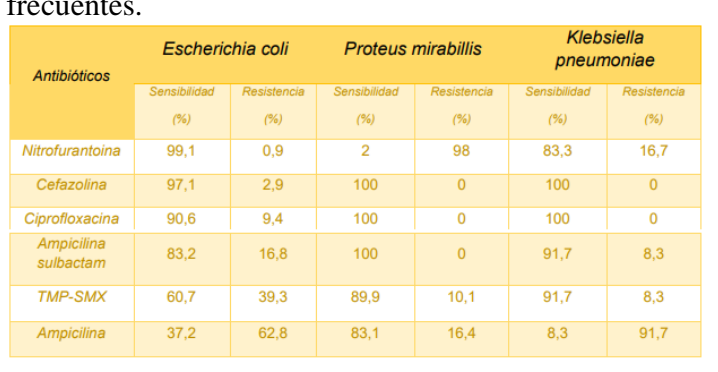

El Proteus mirabillis mostró sensibilidad a cefazolina, ciprofloxacina y ampicilina sulbactam en un $100 \%$, en menor porcentaje a trimetropina sulfametoxazol $(89,9 \%)$ y ampicilina $(83,7 \%)$. Presento resistencia a nitrofurantoina en un $98 \%$ (tabla 1), resistencia natural de la bacteria.

En cuanto a la Klebsiella pneumoniae mostró sensibilidad cefazolina y ciprofloxacina en un $100 \%$, seguido por trimetropina sulfametoxazol y ampicilina sulbactam en un $91,7 \%$ y nitrofurantoina en un $83,3 \%$. Presentó resistencia a la ampicilina en un $91,7 \%$ (Tabla1), resistencia natural de la bacteria.

Con respecto a los otros microorganismos productores de ITU en menor porcentaje se encontró que el Enterococcus faecalis fue multisensible. En cuanto a la Pseudomonas aeruginosa presentó sensibilidad a meropenem y piperacilina tazobactam. 


\section{Discusión}

La ITU es una de las infecciones bacterianas más frecuentes en Pediatría. La edad y el sexo son factores determinantes en la incidencia, siendo más frecuentes en varones menores de 6 meses de edad y en mujeres a partir del año de vida ${ }^{11}$.En nuestro estudio las ITU predominaron en el sexo femenino en todas las edades, aunque se observó que entre el mes y los 3 meses de vida el sexo masculino presentó un porcentaje mayor que en el resto de los grupos etarios sin superar el porcentaje de las mujeres.

El tratamiento empírico inicial de las infecciones urinarias en pediatría es un desafío para los profesionales debido que al momento de iniciarlo no se cuenta con el resultado del urocultivo realizado. Para que un antibiótico pueda ser utilizado en forma inicial no debe tener una tasa de resistencia superior a $10-20 \%{ }^{9}$. Es por ello que es importante conocer en nuestro medio que microorganismo es más frecuente y su perfil de sensibilidad antibiótica.

Al igual que otras series reportadas en nuestro estudio encontramos que la $E$. coli fue el microorganismo más frecuente causal de Infecciones urinarias en todas las edades, seguido por el Proteus mirabillis y la Klebsiella pneumoniae ${ }^{11}$.

Las recomendaciones para el tratamiento empírico de las infecciones urinarias varían según la población que considere. En España se desaconseja el uso inicial de la ampicilina y la amoxicilina debido a la alta resistencia de la E. coli a los mismos ${ }^{11}$.

Además, a causa de los altos porcentajes de resistencia crecientes que presentan las cefalosporinas de $1^{\circ}$ generación y la amoxicilina ácido clavulánico tampoco es recomendable su uso en esta región ${ }^{11}$. En la literatura de nuestro país, basado en la sensibilidad antibiótica de los microrganismos para el tratamiento empírico de las ITU se recomienda el uso de cefalosporinas de $1^{\circ}$ generación, amoxicilina ácido clavulánico o trimetropina sulfametoxazol ${ }^{10}$. Según nuestros resultados, se podría mantener las recomendaciones de uso de cefalosporinas de $1^{\circ}$ generación y amoxicilina ácido clavulanico, ya que los gérmenes más comunes encontrados presentaron alta sensibilidad a estos antibióticos. Por otro lado, la E. coli presentó una alta tasa de resistencia a la trimetropina sulfametoxazol, no así el Proteus mirabillis y la Klebsiella pneumoniae. En cuanto a la ampicilina se encontró una elevada resistencia en la E. coli como se reporta en la literatura $^{11}$.

La nitrofurantoina presentó una sensibilidad alta en infecciones por E. coli y Klebsiella pneumoniae, pero no así en Proteus mirabillis el cual presenta resistencia intrínseca al mismo, por lo que se desaconsejaría su uso, sumado a que no logra una buena concentración en sangre por lo que no sería de primera elección en ITU febriles ${ }^{10}$.

La ciprofloxacina presento una alta sensibilidad en los gérmenes más comunes y en el resto de los gérmenes encontrados, que puede ser a causa de su poco uso por los efectos colaterales en la población pediátrica $^{11}$.

\section{Conclusión}

Nuestro estudio permitió conocer que las infecciones urinarias en nuestro medio en la edad pediátrica son más frecuentes en el sexo femenino y entre el año y los 5 años de vida. El agente etiológico más común es la Escherichia coli, seguido por el Proteus mirabillis y la Klebsiella pneumoniae, los cuales presentan una alta sensibilidad antibiótica a cefazolina, ciprofloxacina y ampicilina sulbactam.

Estos resultados obtenidos permitirían iniciar un tratamiento empírico dirigido y fundamentado.

\section{Bibliografía}

1. Salas del C, P. Actualización en el diagnóstico y manejo de la Infección Urinaria en pediatría. Rev Chil Pediatr. 2012: 269-278.

2. Troche, AV y col. Infección urinaria: un problema frecuente en Pediatría. Revisión de la literatura. Pediatr.; (Asunción). 2018; 45: $165-169$.

3. González Rodríguez, JD y col. Infección de vías urinarias en la infancia. Protoc diagn ter pediatr. 2014; 1:91-108.

4. Hernández, MR y col. Infección urinaria en el niño (1 mes-14 años). Protocolos Diagnóstico Terapeúticos de la AEP: Nefrología Pediátrica. 2008: 54-73. Acceso: https://www.aeped.es/sites/default/files/docu mentos/5_4.pdf.

5. Kutasy, Balazs et al. Urinary Tract Infection in Children: Management in the Era of Antibiotic Resistance-A Pediatric Urologist's View European Urology Focus. 2017;3: $207-211$.

6. M J. F, Dairiki Shortlife L. M. Urinary tract infection in children: etiology and epidemiology. Uro Clin North Am. 2004; 31:517-26. 
7. Braun J. S, Camponovo C. R, Cona T. E. et al. Recomendaciones para el diagnóstico microbiologico de la infección urinaria. Rev Chil Infect. 2001; 18: 57-63.

8. Gonzalo de Liria, CR. Infección Urinaria en Pediatría. En: editor Carlos Pigrau. Infección del tracto urinario. Majadahonda (Madrid). Editorial SALVAT. 2013. 161-169.

9. Troche, A.V. y col. Prevalencia de uropatógenos y sensibilidad antimicrobiana en lactantes menores de 2 años provenientes de la comunidad con diagnóstico de infección de vías urinarias. doi:10.18004/rdn2016.0008.02.034-046.
10. Adragna, M y col. Nuevas recomendaciones frente a las actuales controversias en infección urinaria. Arch Argent Pediatr 2015; 113:57958.

11. Piñeiro Pérez, R. y col. Recomendaciones sobre el diagnóstico y tratamiento de la infección urinaria. An Pediatr (Barc). 2019; 90:400. e1---400.

12. Pezzlo, M. York, M. y col. Clinical Microbiolgy procedures handbook. AmericanSociety for Microbiology Press. Washington DC, 2010: 3.12.1 - 3.12.19. 